\title{
First-principles modeling of magnetic misfit interfaces
}

\author{
Sergiy Grytsyuk and Udo Schwingenschlögl ${ }^{*}$ \\ KAUST, PSE Division, Thuwal 23955-6900, Kingdom of Saudi Arabia
}

(Received 16 August 2013; published 16 October 2013)

\begin{abstract}
We investigate the structural and magnetic properties of interfaces with large lattice mismatch, choosing $\mathrm{Pt} / \mathrm{Co}$ and $\mathrm{Au} / \mathrm{Co}$ as prototypes. For our first-principles calculations, we reduce the lattice mismatch to $0.2 \%$ by constructing Moiré supercells. Our results show that the roughness and atomic density, and thus the magnetic properties, depend strongly on the substrate and thickness of the Co slab. An increasing thickness leads to the formation of a Co transition layer at the interface, especially for Pt/Co due to strong Pt-Co interaction. A Moiré supercell with a transition layer is found to reproduce the main experimental findings and thus turns out to be the appropriate model for simulating magnetic misfit interfaces.
\end{abstract}

DOI: 10.1103/PhysRevB.88.165414

PACS number(s): 68.35.-p, 73.20.-r

\section{INTRODUCTION}

Interfaces between $3 d$ transition metals and noble metals have fruitful features for many technologically important applications. For example, the perpendicular magnetic anisotropy of $\mathrm{Pt} / \mathrm{Co}$ and $\mathrm{Au} / \mathrm{Co}$ interfaces has potential in $\operatorname{logic}^{1,2}$ and memory ${ }^{3}$ devices. Exciting properties (e.g., optical, electrical, and catalytic) of systems built of magnetic and nonmagnetic materials are well known from nanoparticles. ${ }^{4-7}$ Despite their potential for applications, the physical properties of magnetic/nonmagnetic interfaces, such as the origin of the perpendicular magnetic anisotropy, are not well understood, mainly because they depend on external factors such as the interface quality and the film thickness in thin-film geometries. A change of structural details can strongly alter the electronic states, which in turn influences the magnetic properties. For this reason, knowledge about the structure on an atomic level is key for understanding such interfaces.

First-principles techniques based on density functional theory are widely used nowadays for investigating interfaces. It is often assumed that the lattice parameters of the component materials are the same (coherent model). However, this approach is valid only for small lattice mismatch, whereas for the $\mathrm{Au} / \mathrm{Co}$ and $\mathrm{Pt} / \mathrm{Co}$ interfaces, for instance, the lattice mismatch amounts to $16.5 \%$ and $12.7 \%$, respectively, which results in such a strong strain that unphysical results have to be expected. For example, the theoretical study in Ref. 8 is based on ab initio calculations using a coherent model of the $\mathrm{Pt} / \mathrm{Co}$ interface. The authors find for the Co atoms a magnetization of $2.08 \mu_{B}$, while the experimental value is $1.8 \mu_{B}{ }^{9}{ }^{9}$

A possible way to avoid the problem is the application of supercells, in that the two component materials form a Moiré pattern; see Fig. 1. In fact, such patterns have been observed experimentally in a wide range of interfaces, such as graphene/Ir, ${ }^{10,11} \mathrm{Au} / \mathrm{Co},{ }^{12,13} \mathrm{Pt} / \mathrm{Co},{ }^{14} \mathrm{FeO} / \mathrm{Pd},{ }^{15} \mathrm{FeO} / \mathrm{Pt},{ }^{16}$ and $\mathrm{Fe} / \mathrm{MgO} .{ }^{17} \mathrm{By}$ means of Moiré supercells, the strain on the component materials can be reduced significantly. For example, in $(5 \times 5) \mathrm{Au} /(6 \times 6) \mathrm{Co}$ and $(7 \times 7) \mathrm{Pt} /(8 \times 8) \mathrm{Co}$ supercells, the lattice mismatch is reduced to $0.2 \%$. It could be further reduced by using even larger supercells, but with the number of atoms the computational demand of first-principles calculations grows dramatically.

While the supercell approach enables us to describe the physical properties in the bulklike regions properly, the interface itself remains an issue. Does the structure really resemble the bulklike regions, as assumed by standard Moiré supercells? For example, in Ref. 18, it has been demonstrated that the structure of the first two Ni layers at the $\mathrm{CoO} / \mathrm{Ni}$ interface differs significantly from that of bulk Ni and that the electronic and magnetic properties are modified accordingly. The objective of the present work thus is to understand in detail the structures and resulting magnetism at $\mathrm{Au} / \mathrm{Co}$ and $\mathrm{Pt} / \mathrm{Co}$ (111) interfaces as a function of the thickness of the Co slab.

\section{INTERFACE MODEL AND COMPUTATIONAL DETAILS}

The growth of a thin film on a substrate is determined by the interaction between the substrate and the adatoms. If this interaction is stronger than that between the adatoms themselves, the film will tend to resemble the substrate structure. In the opposite case, if the adatom-adatom interaction dominates, the film will aim to maintain a bulklike structure, which leads to the formation of islands (the size of which depends on the lattice mismatch). In the first case, the atomic densities in the substrate and the film can be expected to be the same and the coherent model therefore can be applied. In the second case, in a first approximation, an incoherent structure based on a Moiré pattern will be formed, such that a coherent model will not give correct results. In addition, one has to take into account that the strength of the adatom-adatom interaction can be different for single and few-layer films due to the surface tension. Thus, the structure and atomic density at the interface in general can depend on the substrate (in our case, $\mathrm{Au}$ or $\mathrm{Pt}$ ) and the film thickness.

To investigate the dependencies for the $\mathrm{Au} / \mathrm{Co}$ and $\mathrm{Pt} / \mathrm{Co}$ interfaces, we calculate binding energies for supercells with different Moiré patterns (see Fig. 2), allowing the Co atomic density to be different while the substrate atomic density is fixed to the bulk value. Going beyond the present approach, surface dislocations have been experimentally observed in Refs. 8 and 9. Figure 2 shows that the 5:6 and 7:8 models for the $\mathrm{Au} / \mathrm{Co}$ and $\mathrm{Pt} / \mathrm{Co}$ interfaces, respectively, lead to a Co atomic density similar to the bulk. These models require $25 \mathrm{Au}$ and 36 Co unit cells for $\mathrm{Au} / \mathrm{Co}$ and $49 \mathrm{Pt}$ and $64 \mathrm{Co}$ unit cells for $\mathrm{Pt} / \mathrm{Co}$ to reduce the lattice mismatch to $0.2 \%$. In the coherent model, the Co atomic density would be too small to derive realistic results. Since the incoherent models contain a huge number 


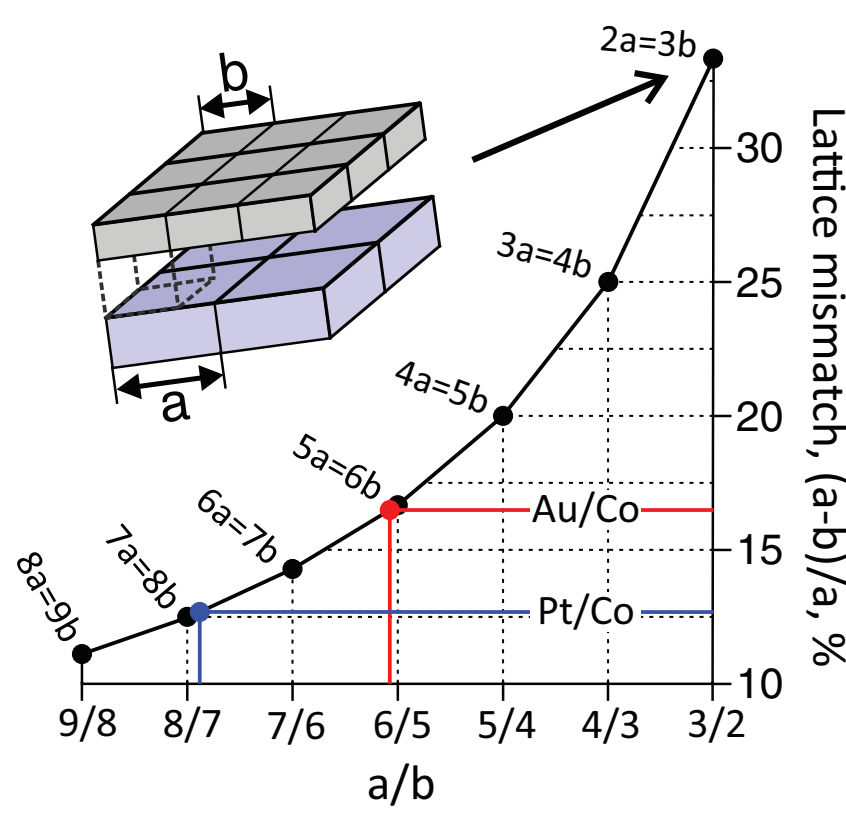

FIG. 1. (Color online) Relationship between lattice mismatch and Moiré pattern. Red and blue circles indicate the lattice mismatch of the $\mathrm{Au} / \mathrm{Co}$ and $\mathrm{Pt} / \mathrm{Co}$ interfaces, respectively. Application of $(5 \times 5) \mathrm{Au} /(6 \times 6) \mathrm{Co}$ and $(7 \times 7) \mathrm{Pt} /(8 \times 8) \mathrm{Co}$ supercells reduces the lattice mismatch to $0.2 \%$.

of atoms, we perform calculations for periodically repeated slabs of three substrate layers ( $\mathrm{Au}$ or $\mathrm{Pt}$ ) and one or four $\mathrm{Co}$ layers on top. The bottom of the substrate is terminated by $\mathrm{H}$ atoms and a vacuum layer of $10 \AA$ width follows the Co film. In Figs. 3(a) and 3(b), respectively, the coherent model and an example of an incoherent model are presented. The other models used in our calculations have similar structures at the interface with on-top, hollow, and some intermediate Co sites, with respect to the substrate.

If the adatom-adatom and adatom-substrate interactions are of similar strength, we expect the formation of a transition layer

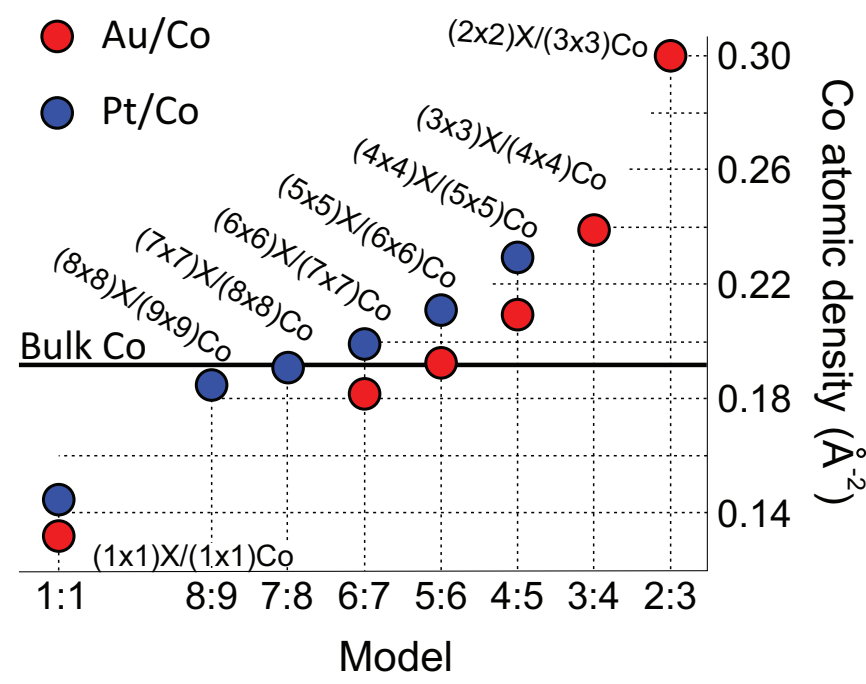

FIG. 2. (Color online) Dependence of the Co atomic density on the size of the $X /$ Co supercell $(X=\mathrm{Au}, \mathrm{Pt})$. The atomic density of $X$ is fixed. A horizontal line indicates the atomic density in bulk Co.

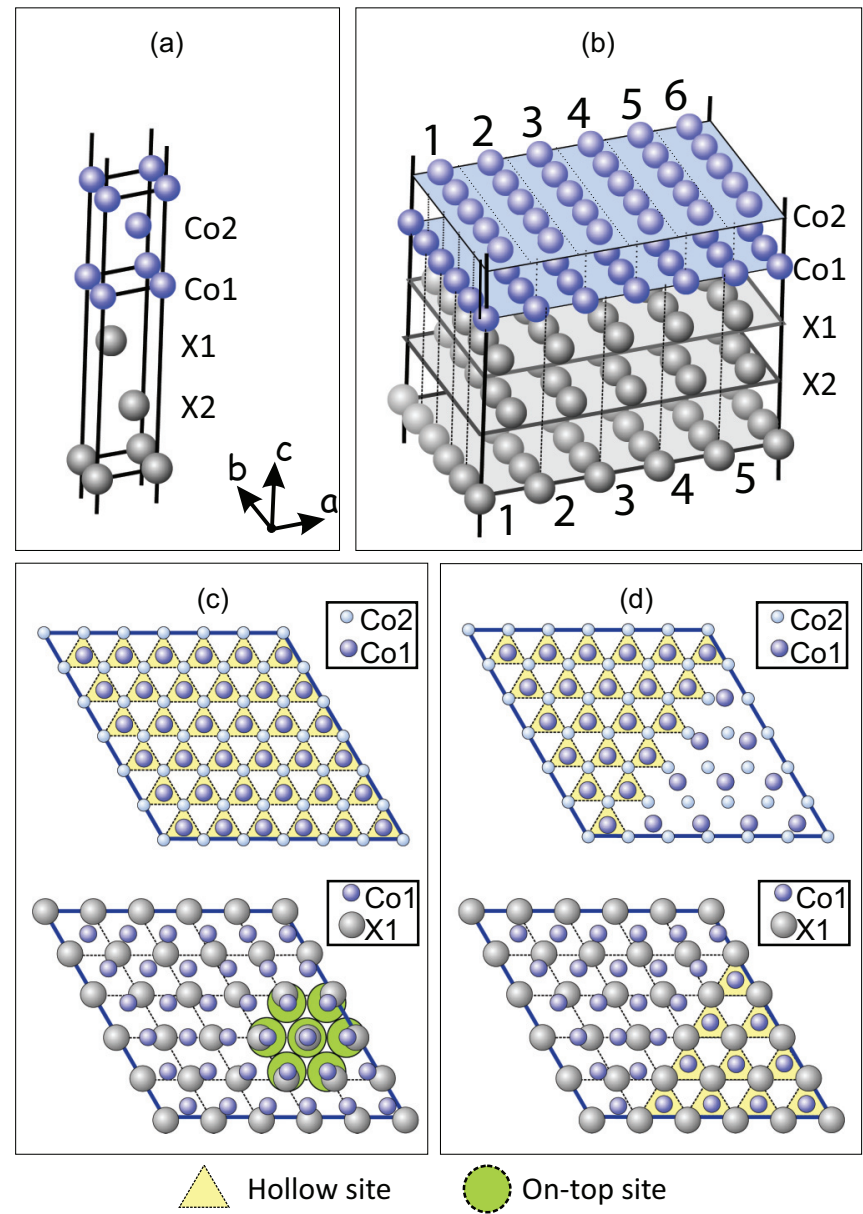

FIG. 3. (Color online) $X / \mathrm{Co}$ interfaces $(X=\mathrm{Pt}, \mathrm{Au})$ : (a) Coherent model. The atomic densities of $X$ and Co are the same (atoms of the Co1 layer occupy hollow sites of the $X 1$ layer). (b) Supercell containing $5 \times 5 X$ and $6 \times 6$ Co unit cells per layer; top views are shown in (c) and (d). (c) The interface Co1 layer has the same structure as the bulk (atoms of the Co1 layer occupy hollow sites of the $\mathrm{Co} 2$ layer). (d) The interface $\mathrm{Co} 1$ layer constitutes a transition layer between the substrate $X 1$ layer and next Co2 layer (atoms of the Co1 layer occupy partially hollow sites of the $X 1$ layer and partially hollow sites of the Co2 layer).

that partially reflects the structure of the substrate and partially that of bulk Co. It has been shown in Ref. 18 that shifting of Co atoms from on-top sites [see Fig. 3(c)] to hollow sites [see Fig. 3(d)] increases the binding energy (lowers the total energy) significantly. Such a layer is called the transition layer and reduces the strain energy. To check this concept for the $\mathrm{Au} / \mathrm{Co}$ and $\mathrm{Pt} / \mathrm{Co}$ interfaces, we modify the first Co layer in all the above-mentioned models, as shown in Fig. 3(d) for the $(5 \times$ $5) X /(6 \times 6)$ Co model. The Co atoms in the transition layer partially occupy the hollow sites of the substrate and partially occupy the hollow sites of the next Co layer, while without the transition layer, they would occupy only Co hollow sites.

We employ the plane-wave pseudopotential VASP (Vienna $A b$ initio Simulation Package, version 5.2.11) in which the Kohn-Sham equations are self-consistently solved by iterative diagonalization and density mixing. For the exchange correlation potential, we use the generalized gradient approximation in the Perdew-Burke-Ernzerhof flavor. Tests have been 


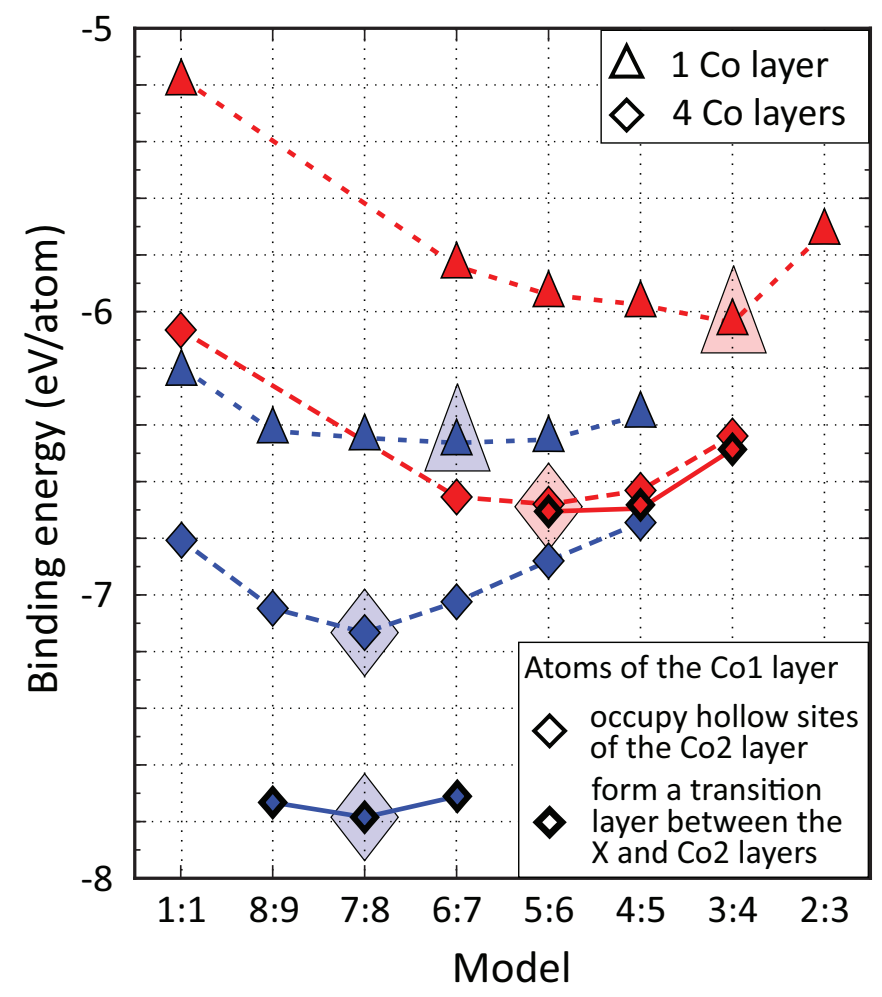

FIG. 4. (Color online) Dependence of the average binding energy of the Co atoms on the size of the supercell. For more details about the models, see Fig. 2. Big shaded triangles (diamonds) indicate the most stable models with one (four) Co layer(s).

performed for $(3 \times 3) \mathrm{Au} /(4 \times 4) \mathrm{Co}$ and $(5 \times 5) \mathrm{Pt} /(6 \times 6) \mathrm{Co}$ supercells with different $k$ meshes to certify that $6 \times 6 \times 1$ and $3 \times 3 \times 1 k$ meshes, respectively, are sufficient (differences of about $1 \mathrm{meV})$.

\section{RESULTS AND DISCUSSION}

According to Figs. 2 and 4 , the Co structure is very similar to the bulk in the $(5 \times 5) \mathrm{Au} /(6 \times 6) \mathrm{Co}$ and $(7 \times 7) \mathrm{Pt} /(8 \times 8) \mathrm{Co}$ interfaces (5:6 and 7:8 models). To address the stability as well as the modifications when more Co layers are added, we calculate the average binding energy of the Co atoms for all models,

$$
E_{B}=\frac{1}{N}\left(E_{X / \mathrm{Co}}-E_{X}\right)-E_{\mathrm{Co}},
$$

where $E_{X / \mathrm{Co}}, E_{X}$, and $E_{\mathrm{Co}}$ are the total energies of the full interface, the $X$ substrate, and a single Co atom, respectively. Moreover, $N$ is the number of Co atoms. The results are summarized in Fig. 4: Coherent models with one or four Co layers on top of the substrate are unstable due to the large induced strain in the Co region, especially for the $\mathrm{Au} / \mathrm{Co}$ interface. The Co-Pt coupling is stronger than the Co-Au coupling and the structures are different for one and four Co layers. Adding Co layers modifies the optimal Moiré patterns from $(3 \times 3) \mathrm{Au} /(4 \times 4) \mathrm{Co}$ to $(5 \times 5) \mathrm{Au} /(6 \times 6) \mathrm{Co}$ and from $(6 \times 6) \mathrm{Pt} /(7 \times 7) \mathrm{Co}$ to $(7 \times 7) \mathrm{Pt} /(8 \times 8) \mathrm{Co}$.

In the case of one Co layer, the Co atomic density is increased slightly for the Pt substrate (3\%) and significantly for
TABLE I. Co atomic density at the interface (in $\AA^{-2}$ ) and surface roughness (in $10^{-3} \AA^{-1}$ ) for the ground-state structures shown in Fig. 4 by shaded triangles and diamonds. An asterisk indicates the presence of a transition layer.

\begin{tabular}{lccccc}
\hline \hline & \multicolumn{2}{c}{ Atomic density } & & \multicolumn{2}{c}{ Roughness } \\
\cline { 2 - 3 } \cline { 5 - 6 } Film thickness & $\mathrm{Au} / \mathrm{Co}$ & $\mathrm{Pt} / \mathrm{Co}$ & & $\mathrm{Au} / \mathrm{Co}$ & $\mathrm{Pt} / \mathrm{Co}$ \\
\hline 1 layer of Co & 0.237 & 0.199 & & 6.72 & 0.7 \\
4 layers of Co & 0.193 & 0.191 & & 0.26 & 0.19 \\
4 layers of Co* & 0.160 & 0.167 & & 0.11 & 0.05 \\
Bulk Co & \multicolumn{3}{c}{0.192} & & \\
\hline \hline
\end{tabular}

the Au substrate (23\%). This strong compression is explained by the weak Au-Co coupling. In the case of four Co layers, the most stable models have Co atomic densities close to the bulk value. Moreover, a Co transition layer enhances the binding energy significantly for the Pt substrate due to strong Co-Co and Co-Pt coupling. Because of the latter, the interface $\mathrm{Co}$ atoms occupy energetically favorable sites with respect to both the neighboring Pt and Co layers. For the Au substrate, the $\mathrm{Co}-\mathrm{Au}$ interaction is weak and the Co structure is optimized independently, such that only hollow sites of the adjacent Co layer are occupied. The dependence of the interface Co atomic density on the substrate and number of Co layers is addressed in Table I. In the case of one Co layer, it is higher than in bulk Co, while adding three extra layers reduces it due to the formation of a transition layer.

Our next goal is to understand how the Co surface roughness depends on the substrate and number of Co layers. The roughness can be estimated as the average shift of the surface atoms in the vertical direction:

$$
R=\frac{1}{n A} \sum|\Delta z|,
$$

where $\Delta z$ is the difference between the average of the vertical positions of all atoms and of the vertical position of a given adatom, $n$ is the number of adatoms in the supercell, and $A$ is the area of the basal plane of the supercell. Results are given for the most stable structures in Table I, and graphical illustrations of the surface roughness are shown in Fig. 5. A huge surface roughness is present in the case of one Co layer attached to $\mathrm{Au}$ (almost 10 times higher than for one $\mathrm{Co}$ layer attached to $\mathrm{Pt}$ ) because the $\mathrm{Co}$ atomic density is large (Table I) due to the weak Au-Co interaction. Keeping the Co atoms in a plane thus would cost much in terms of energy. Increasing the number of Co layers for both substrates to four decreases the Co atomic density at the interface and the surface becomes smoother; compare Fig. 5. The roughness decreases dramatically by about 26 times in the Au/Co case, while in the $\mathrm{Pt} / \mathrm{Co}$ case, it decreases only by four times.

Moreover, the presence of a Co transition layer at the interface decreases not only the interface Co atomic density, but also the Co surface roughness (Table I): For Pt/Co, the surface becomes smoother by four times and for $\mathrm{Au} / \mathrm{Co}$ by two times, as compared to the situation without a transition layer. This is another reason why the transition layer stabilizes the structure more in the former than in the latter case. In the experimental Refs. 12 and 13, for $\mathrm{Au} / \mathrm{Co}$, it was shown that 

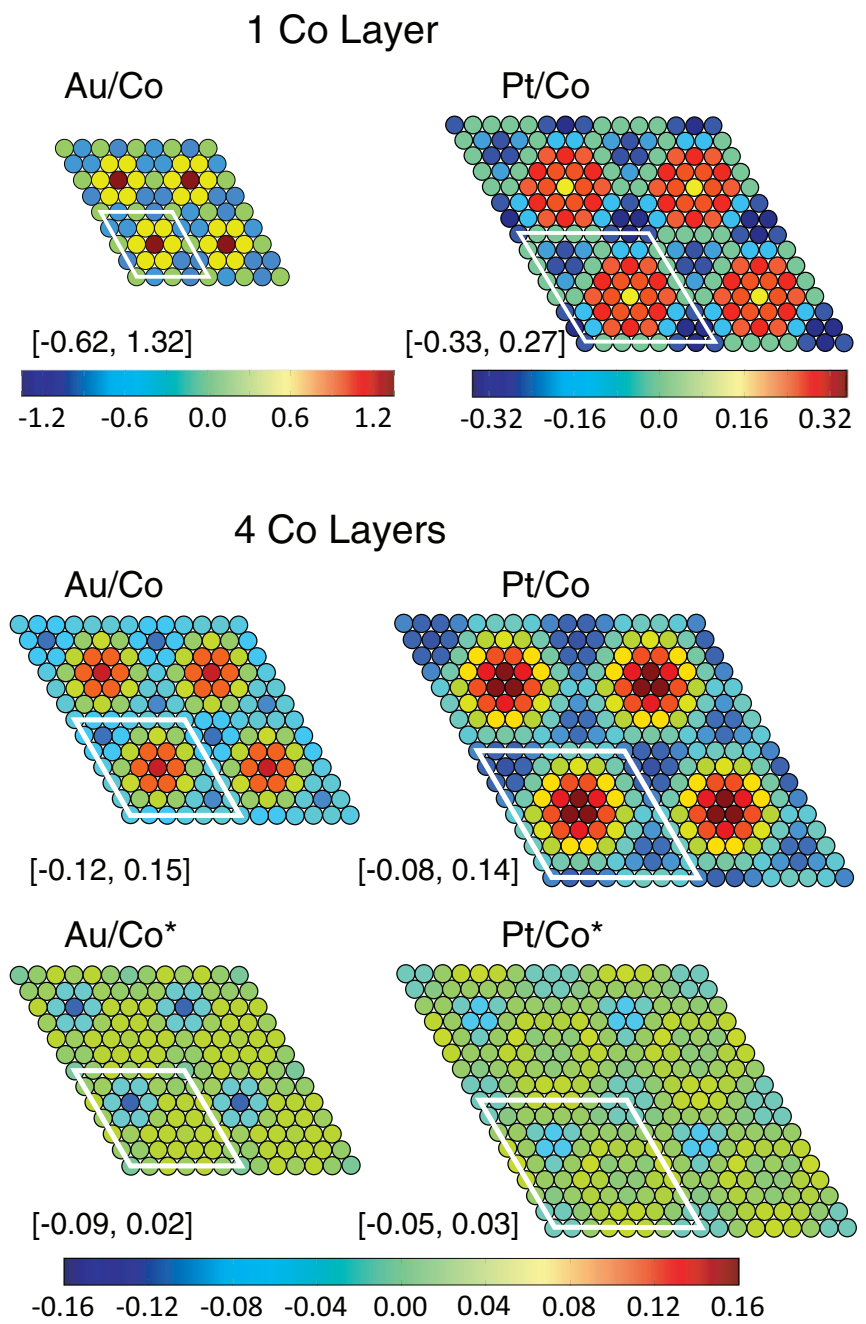

FIG. 5. (Color online) Roughness of the Co surface for one and four Co layers on $\mathrm{Au}$ or Pt. The numbers in brackets are the maximal deviations of Co atoms from the average $z$ value. An asterisk indicates the presence of a transition layer.

the Co corrugation $\left(z_{\max }-z_{\min }\right)$ is $1.75 \AA$, which is close to our calculated value of $1.94 \AA$. In addition, the experiments in Ref. 14 for the Pt/Co interface indicate that the periodicity of the Moiré pattern is $20 \AA$; we obtain $19.6 \AA$.

Since the structure depends on the substrate and number of Co layers, the magnetic properties are expected to be different as well. In Fig. 6, we present the average magnetization per layer for the coherent and most stable incoherent models. While the magnetic properties of the noble metals are, as expected, hardly different, the behavior of Co depends significantly on the atomic density. First, we notice that the coherent model in each case gives too high magnetic moments, by $0.26(0.22) \mu_{B}$ for $\mathrm{Au} / \mathrm{Co}$ and by $0.19(0.16) \mu_{B}$ for Pt/Co in the case of one (four) Co layer(s). Our calculated average magnetic moments of $1.83 \mu_{B}$ for the interface Co1 layer and of $1.64 \mu_{B}$ for the bulklike $\mathrm{Co} 2$ layer of the Pt/Co system are very close to the experimental values $\left(1.8 \mu_{B}\right.$ and $1.58 \mu_{B}$, respectively). ${ }^{9}$ Due to spin polarization of the interface $\mathrm{Pt}$ atoms, the interface $\mathrm{Co}$ atoms in $\mathrm{Pt} / \mathrm{Co}$ have higher magnetic moments than in $\mathrm{Au} / \mathrm{Co}$. Adding Co layers decreases the magnetic moment at the interface if no transition layer is (a)
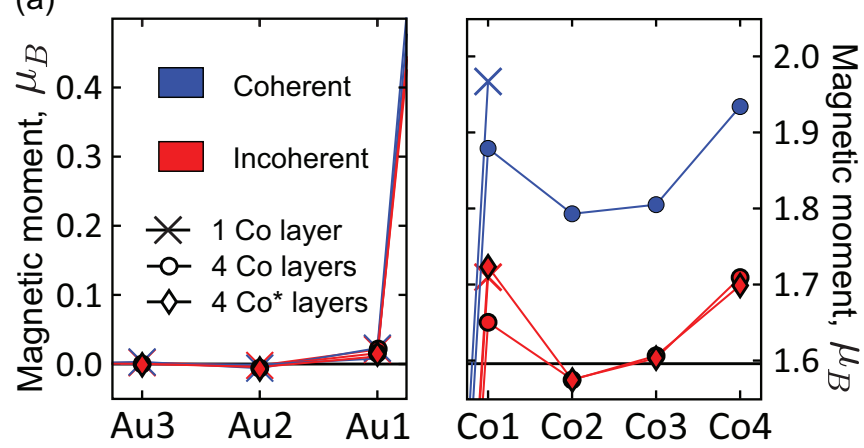

(b)
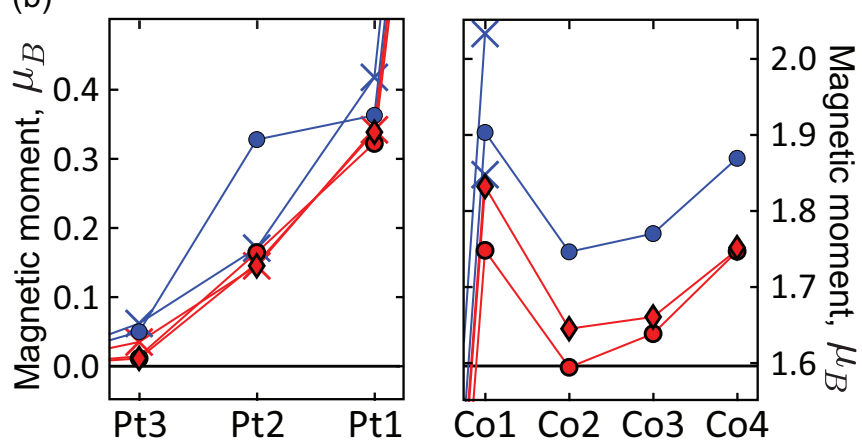

FIG. 6. (Color online) Average magnetic moment per layer for the (a) $\mathrm{Au} / \mathrm{Co}$ interface and (b) Pt/Co interface. Vertical black lines indicate the magnetization in the bulk compounds. An asterisk indicates the presence of a transition layer. Au1, Pt1, and Co1 are the interface layers.

formed, while formation of such a layer decreases the Co atomic density and thus enhances the magnetization.

\section{CONCLUSION}

In conclusion, the lattice mismatch between $\mathrm{Co}$ and $\mathrm{Au}$ or Pt modifies the structure and magnetic properties of an interface significantly, demonstrating that the coherent model will not give valid results. The same applies to the standard supercell approach with constant atomic density in the Co slab. We therefore propose a model based on a Moiré pattern that includes a Co transition layer at the interface and demonstrate that it gives results very close to the experimental situation. Depending on the substrate and thickness of the Co film, the Co atomic density near the interface is different. While the atomic density and surface roughness for a single Co layer on top of $\mathrm{Au}$ are found to be large, due to the weak $\mathrm{Au}-\mathrm{Co}$ and strong $\mathrm{Co}-\mathrm{Co}$ interaction, additional Co layers result in lower atomic density and surface roughness. A very similar thickness dependence is observed for $\mathrm{Pt} / \mathrm{Co}$, for which an increasing thickness additionally causes the formation of a transition layer. In this layer, the atoms partially occupy energetically favorable sites of both the neighboring $\mathrm{Pt}$ and Co layers. Formation of a transition layer in $\mathrm{Au} / \mathrm{Co}$ results only in a small stability gain, since the Au-Co interaction is weaker than the CoCo interaction. As the interface magnetic properties depend substantially on the employed model, our result demonstrates that a careful selection is critical. The proposed Moiré supercell with transition layer turns out to give excellent results. 
*udo.schwingenschlogl@kaust.edu.sa

${ }^{1}$ D. A. Allwood, G. Xiong, C. C. Faulkner, D. Atkinson, D. Petit, and R. P. Cowburn, Science 309, 1688 (2005).

${ }^{2}$ P. Xu, K. Xia, C. Gu, L. Tang, H. Yang, and J. Li, Nat. Nanotech. 3, 97 (2008).

${ }^{3}$ S. S. P. Parkin, M. Hayashi, and L. Thomas, Science 320, 190 (2008). ${ }^{4}$ S. Sao-Joao, S. Giorgio, J. M. Penisson, C. Chapon, S. Bourgeois, and C. Henry, J. Phys. Chem. B 109, 342 (2005).

${ }^{5}$ J. Zhang, F. H. B. Lima, M. H. Shao, K. Sasaki, J. X. Wang, J. Hanson, and R. R. Adzic, J. Phys. Chem. B 109, 22701 (2005).

${ }^{6} \mathrm{Z}$. Xu, Y. Hou, and S. Sun, J. Am. Chem. Soc. 129, 8698 (2007).

${ }^{7}$ F. Bao, J.-F. Li, B. Ren, J.-L. Yao, R.-A. Gu, and Z.-Q. Tian, J. Phys. Chem. C 112, 345 (2008).

${ }^{8}$ A. Lehnert, S. Dennler, P. Bloński, S. Rusponi, M. Etzkorn, G. Moulas, P. Bencok, P. Gambardella, H. Brune, and J. Hafner, Phys. Rev. B 82, 094409 (2010).

${ }^{9}$ G. Moulas, A. Lehnert, S. Rusponi, J. Zabloudil, C. Etz, S. Ouazi, M. Etzkorn, P. Bencok, P. Gambardella, P. Weinberger, and H. Brune, Phys. Rev. B 78, 214424 (2008).
${ }^{10}$ L. Meng, R. T. Wu, L. Z. Zhang, L. F. Li, S. X. Du, Y. L. Wang, and H. J. Gao, J. Phys.: Condens. Matter 24, 314214 (2012).

${ }^{11}$ R. Decker, J. Brede, N. Atodiresei, V. Caciuc, S. Blügel, and R. Wiesendanger, Phys. Rev. B 87, 041403 (2013).

${ }^{12}$ L. Cagnon, A. Gundel, T. Devolder, A. Morrone, C. Chappert, J. E. Schmidt, and P. Allongue, Appl. Surf. Sci. 164, 22 (2000).

${ }^{13}$ L. Cagnon, T. Devolder, R. Cortes, A. Morrone, J. E. Schmidt, C. Chappert, and P. Allongue, Phys. Rev. B 63, 104419 (2001).

${ }^{14}$ P. Grütter and U. T. Dürig, Phys. Rev. B 49, 2021 (1994).

${ }^{15}$ S. K. Shaikhutdinov, R. Meyer, D. Lahav, M. Bäumer, T. Klüner, and H.-J. Freund, Phys. Rev. Lett. 91, 076102 (2003).

${ }^{16}$ M. Ritter, W. Ranke, and W. Weiss, Phys. Rev. B 57, 7240 (1998).

${ }^{17}$ G. X. Miao, J. Y. Chang, M. J. van Veenhuizen, K. Thiel, M. Seibt, G. Eilers, M. Munzenberg, and J. S. Moodera, Appl. Phys. Lett. 93, 142511 (2008).

${ }^{18}$ S. Grytsyuk, M. V. Peskov, and U. Schwingenschlögl, Phys. Rev. B 86, 174115 (2012). 